\title{
Prevención y gestión del dolor musculoesquelético en el personal de enfermería
}

\author{
Prevention and management of musculoskeletal pain \\ in nursing staff
}

\author{
Mercè Soler-Font ${ }^{1}$ \\ ${ }^{1}$ Centre d'Investigació en Salut Laboral (CISAL), Barcelona, España.
}

Fechas · Dates

Recibido: 2020.07.19

Publicado: 2020.11.04
Sección coordinada por / Section Coordinator

Dr. Guillermo García González

Profesor Titular Derecho del Trabajo y SS Universidad Internacional de La Rioja (UNIR)

Email: guillermo.garcia@unir.net 


\section{Entrevista a Mercè Soler-Font}

1. ¿Cuál es la procedencia, especialización y áreas de trabajo de los autores del artículo? ¿Qué características le parecen más destacables de este grupo de trabajo?

Los autores de este artículo provenimos de diferentes profesiones incluyendo médicos, fisioterapeutas, epidemiólogos y estadísticos. Todos los autores nos movemos en las áreas de salud pública, salud laboral y evaluación de intervenciones, y la mayoría somos doctores o estamos haciendo el doctorado. Lo que me parece más destacable de este grupo de trabajo es que es multidisciplinar e internacional, lo cual nos permite aprender de otros equipos y complementar los diferentes puntos de vista.

\section{2. ¿Cómo se ha financiado el estudio?}

Este estudio ha sido financiado por dos fondos del Instituto de Salud Carlos III-FEDER (PI14/01959 y PI17/00779), y recibió también una beca de la Fundación Prevent, lo que nos permitió desarrollar una parte de la intervención, en concreto la promoción de la salud.

\section{3. ¿Qué problema pretende abordar este estudio y dónde radica su interés o relevancia para la seguridad y salud en el trabajo?}

Este proyecto parte de la necesidad de reducir y gestionar el dolor musculoesquelético en los trabajadores, ya que alrededor del $70-80 \%$ de los trabajadores en Europa refieren tener algún tipo de dolor o molestia, y concretamente en el personal sanitario español este porcentaje llega a superar el $80 \%$. En este sentido consideramos muy importante mejorar la salud osteomuscular de los trabajadores y la discapacidad asociada a ésta, desde una perspectiva biopsicosocial dado el origen multicausal del dolor.

\section{4. ¿Qué aporta este estudio de novedoso o destacable en relación al resto de producción científica sobre el problema estudiado?}

Hay tres aspectos destacables en este estudio, el primero es que partimos del modelo biopsicosocial de la salud y consecuentemente tenemos una intervención multidisciplinar. El segundo aspecto es que no solamente se centra en gestionar el dolor musculoesquelético, sino que también pretende prevenir su aparición. Y en tercer lugar, y creo que el más importante, es que para lograr esta atención integral, abordamos los tres niveles de prevención, es decir prevención primaria, para prevenir la aparición del dolor, secundaria, creando una vía de detección y atención precoz, y terciaria, tratando a las personas que ya padecen alguna dolencia.

\section{5. ¿Se han encontrado con alguna dificultad o contratiempo para el desarrollo del estudio?}

Implementar una intervención de estas características en el lugar de trabajo no era tarea fácil, si bien a medida que avanzábamos fuimos aprendiendo y superando las dificultades. En este sentido, contamos con una figura clave para el proyecto, la champion, que coordinó y dinamizó la implementación del estudio. Además, 
contamos con el apoyo de los hospitales, de los servicios de salud laboral, de los expertos que ofrecían las distintas actividades de la intervención, de las coordinadoras de enfermería, de las supervisoras de las unidades, y de los profesionales referentes o voluntarios de las unidades de intervención que en todo momento tuvieron una actitud muy buena y predisposición a ayudar en el desarrollo de la intervención.

\section{6. ¿Se han obtenido los resultados esperados o se ha producido alguna sorpre- sa?}

En nuestra hipótesis esperábamos ver una reducción del 25\% del dolor en el grupo control versus el grupo intervención, y finalmente hubo un 63\% menos de riesgo en dolor cervical, dorsal y de hombros, así que en este aspecto nos sorprendimos positivamente. Pero, por otro lado, no encontramos diferencias estadísticamente significativas en dolor lumbar, aunque sí que se observó una clara tendencia, ni en incapacidad temporal asociada.

\section{7. ¿Cómo ha sido el proceso de publicación? ¿Han sufrido alguna incidencia?}

De momento hemos publicado el protocolo y la efectividad del estudio sin ninguna incidencia, aunque sí que al ser un ensayo clínico nos han requerido varios documentos y datos adicionales como el protocolo de estudio, las bases de datos y el registro del estudio. Y actualmente, estamos trabajando en los manuscritos de la evaluación de proceso y económica.

\section{8. ¿Qué implicaciones tiene este estudio para la prevención de riesgos labora- les? ¿Cuál sería la recomendación para mejorar la práctica profesional en rela- ción al problema estudiado?}

La intervención del proyecto INTEVAL_Spain se caracteriza por su flexibilidad, eficiencia y capacidad de adaptarse a las necesidades de las diferentes empresas. Además, está diseñado para optimizar y aprovechar al máximo los recursos de prevención existentes en la empresa. Por lo tanto, sería genial poder implementarla en más hospitales; de hecho, este proyecto empezó en el Parc de Salut Mar y Corporació Sanitaria Parc Taulí, y actualmente se han incorporado dos hospitales más, la Corporació Sanitaria del Maresme y el IAS de Girona, así como otros entornos laborales.

La recomendación principal para mejorar la práctica profesional en relación con el dolor musculoesquelético es que las acciones a desarrollar deberían centrarse en intervenciones multicomponentes, no medicalizadas y que abarquen los tres niveles de prevención. Y también, que no deben ser acciones puntuales, sino que sean sostenibles a lo largo del tiempo. 


\section{Resumen del artículo(1)}

\section{Introducción}

Entre un 70 y $80 \%$ de los trabajadores en Europa refieren tener algún tipo de dolor musculoesquelético. En España, más del 80\% del personal de enfermería (enfermero/as y auxiliares) sufren este tipo de dolencias. Se han desarrollado intervenciones anteriores para reducir el dolor musculoesquelético y mejorar el retorno al trabajo, sin embargo se necesitan enfoques multicomponentes y una evaluación exhaustiva. Este estudio tuvo como objetivo evaluar una intervención multifacética para prevenir y gestionar el dolor musculoesquelético en dos hospitales.

\section{Métodos}

Realizamos un ensayo controlado aleatorizado por clústeres, con un grupo control de intervención tardía. Los clústeres eran unidades hospitalarias independientes con el personal de enfermería como participantes. La intervención abarcó tres componentes basados en la evidencia científica disponible: ergonomía participativa (prevención primaria), actividades de promoción de la salud (prevención primaria) y gestión de casos (prevención secundaria y terciaria). Tanto el grupo intervención como el control recibieron la atención habitual de salud laboral. La intervención duró un año. El dolor musculoesquelético y los datos de capacidad funcional en el trabajo se recogieron al inicio del estudio, a los seis y a los doce meses de seguimiento. Se calcularon las odds ratio (OR) y sus intervalos de confianza del 95\% (IC del 95\%) para el riesgo de dolor musculoesquelético en el grupo de intervención en comparación con el grupo de control mediante regresión logística a través de GEE. Las diferencias en la capacidad funcional en el trabajo entre el grupo de intervención y control se analizaron mediante regresión lineal a través de GEE. La incidencia de incapacidad temporal se calculó a través de la regresión logística y se usó un modelo de riesgo proporcional de Cox para analizar el efecto de la intervención sobre la duración de la incapacidad temporal.

\section{Resultados}

Ocho clústeres fueron aleatorizados, incluyendo 473 enfermeras y auxiliares de enfermería. A los doce meses, el grupo de intervención mostró una disminución estadísticamente significativa del riesgo de dolor de cuello, hombros y espalda cervical y dorsal, en comparación con el grupo control $(\mathrm{OR}=0,37$; IC del $95 \%=0,14$ a 0,96). También se observó una reducción del dolor lumbar no estadísticamente significativa. No encontramos diferencias en la capacidad funcional ni en la incidencia y duración de la incapacidad temporal. 


\section{Conclusiones}

La intervención fue efectiva para reducir el dolor de cuello, hombro y espalda cervical y dorsal. Nuestros resultados, sugieren que las intervenciones para prevenir y gestionar el dolor musculoesquelético necesitan un enfoque multifactorial que incluya los tres niveles de prevención, y que estén enmarcados dentro del modelo biopsicosocial.

\section{Referencias}

1. Soler-Font M, Ramada JM, van Zon SK, Almansa J, Bültmann U, Serra C. Multifaceted intervention for the prevention and management of musculoskeletal pain in nursing staff: Results of a cluster randomized controlled trial. PLoS One. 2019;14(11):e0225198. doi:10.1371/journal.pone.0225198. 